University of New Orleans

ScholarWorks@UNO

$11-2011$

\title{
Poincaré sphere representation of the fixed-polarizer rotating- retarder optical system
}

\author{
R. M.A. Azzam \\ University of New Orleans, razzam@uno.edu
}

Follow this and additional works at: https://scholarworks.uno.edu/ee_facpubs

Part of the Electrical and Electronics Commons, and the Optics Commons

\section{Recommended Citation}

R. M. A. Azzam, "Poincaré sphere representation of the fixed-polarizer rotating-retarder optical system," J. Opt. Soc. Am. A 17, 2105-2107 (2000)

This Article is brought to you for free and open access by the Department of Electrical Engineering at ScholarWorks@UNO. It has been accepted for inclusion in Electrical Engineering Faculty Publications by an authorized administrator of ScholarWorks@UNO. For more information, please contact scholarworks@uno.edu. 


\title{
Poincaré sphere representation of the fixed- polarizer rotating-retarder optical system
}

\author{
R. M. A. Azzam \\ Department of Electrical Engineering, University of New Orleans, New Orleans, Louisiana 70148
}

Received June 16, 2000; revised manuscript received July 21, 2000; accepted July 26, 2000

\begin{abstract}
The trajectory of the polarization state of a monochromatic light beam after it passes through a fixed linear polarizer and a rotating linear retarder of arbitrary retardance $\Delta$ is determined on the Poincaré sphere. The three-dimensional figure- 8 contour is shown to be the line of intersection of a right-circular cylinder with the sphere. The cylinder is parallel to the polar $\left(s_{3}\right)$ axis, touches the sphere at the equator (at the point that represents the linear polarization transmitted by the fixed polarizer), and has a radius $r=\sin ^{2}(\Delta / 2)$. Projections of the trajectory in the coordinate planes of the normalized Stokes parameter space $\left(s_{1}, s_{2}, s_{3}\right)$ are also determined. (C) 2000 Optical Society of America [S0740-3232(00)02411-X]
\end{abstract}

OCIS codes: 260.5430, 120.2130, 120.4640, 230.0230.

The combination of a linear polarizer and linear retarder is a versatile optical system that is commonly used for polarization-state generation (PSG) and polarizationstate detection ${ }^{1-4}$ (PSD). Rotation of these elements around the light beam axis creates a variable elliptic polarizer or analyzer. The retardance (differential phase shift) $\Delta$ of the retarder is often selected as quarter wave $\left(\Delta=90^{\circ}\right)$. This choice is essential for complete PSG, to produce all possible polarization states (including the right- and left-circular polarizations) that correspond to all points on the surface of the Poincare sphere. ${ }^{5-7}$ However, it has been shown recently ${ }^{8}$ that for PSD, other choices of $\Delta$ may be optimal $\left(\Delta=132^{\circ}\right)$.

When the polarizer is set at a fixed azimuth and the retarder is rotated, the point that represents the emergent polarization state in PSG (or the elliptic analyzer in PSD) traces a three-dimensional, nonplanar, figure-8 contour on the Poincaré sphere. A sketch of such a contour on the Poincaré sphere for a rotating quarter-wave retarder (QWR) appears in an early paper by Rajagopolan and Ramaseshan. ${ }^{9} \quad$ A computer-generated family of such curves, for different values of $\Delta$, are presented by Sabatke et $a l{ }^{8}$ Stereographic projections of the QWR contour onto a tangent (complex) plane are given by Azzam et $a l .{ }^{3,10}$

In this communication, the exact nature of this contour is determined as the line of intersection of a right-circular cylinder with the Poincaré sphere. The cylinder axis is parallel to the polar $\left(s_{3}\right)$ axis, touches the sphere at the equator (at the point that represents the linear polarization transmitted by the fixed polarizer), and has a radius $r=\sin ^{2}(\Delta / 2)$. (Thus $r=0,1 / 2$, and 1 , when $\Delta=0, \pi / 2$, and $\pi$, respectively.) Normal projections of the trajectory in the coordinate planes of the normalized Stokes parameter space are also determined.

Figure 1 shows a PSG system that consists of an ideal linear polarizer with transmission-axis azimuth $P$ and a linear retarder of retardance $\Delta$ and fast-axis azimuth $C$. $P$ and $C$ are measured from the $x$ axis of a transverse $x y$ reference coordinate system for a monochromatic light beam traveling in the direction of the $z$ axis. When the polarizer is fixed, we set $P=0$ for simplicity and without loss of generality. With the Stokes-Mueller calculus, ${ }^{1,3}$ the normalized Stokes parameters of the output light are obtained as

$$
\begin{aligned}
& s_{0}=1, \quad s_{1}=\cos ^{2} 2 C+\sin ^{2} 2 C \cos \Delta, \\
& s_{2}=\sin 2 C \cos 2 C(1-\cos \Delta), \quad s_{3}=\sin 2 C \sin \Delta .
\end{aligned}
$$

The normalized Stokes vector $\mathbf{s}=\left(s_{1}, s_{2}, s_{3}\right)$ is of unit length, and its tip traces a figure- 8 contour on the Poincaré sphere as the retarder is rotated (and $C$ is changed) for a given $\Delta$. To reveal the nature of this contour, the normalized Stokes parameters $s_{1}$ and $s_{2}$ are rewritten in the following form

$$
\begin{aligned}
& s_{1}=\cos ^{2}(\Delta / 2)+\sin ^{2}(\Delta / 2) \cos 4 C, \\
& s_{2}=\sin ^{2}(\Delta / 2) \sin 4 C .
\end{aligned}
$$

$C$ is readily eliminated between Eqs. (2a) and (2b) so that

$$
\left[s_{1}-\cos ^{2}(\Delta / 2)\right]^{2}+s_{2}{ }^{2}=\sin ^{4}(\Delta / 2) .
$$

Equation (3), which represents the normal projection of the contour in the equatorial $\left(s_{1}, s_{2}\right)$ plane, is that of a circle with center at

$$
\left(s_{1}, s_{2}\right)=\left[\cos ^{2}(\Delta / 2), 0\right],
$$

and radius of

$$
r=\sin ^{2}(\Delta / 2) .
$$

It follows that the trajectory of the polarization state at the output of the fixed-polarizer rotating-retarder system of Fig. 1 is the line of intersection with the Poincaré sphere of a right-circular cylinder whose axis and radius are given by Eqs. (4) and (5), respectively.

Figure 2 shows the cylinder-sphere intersection for QWR $\left(\Delta=90^{\circ}\right)$. For this retardance value the cylinder 


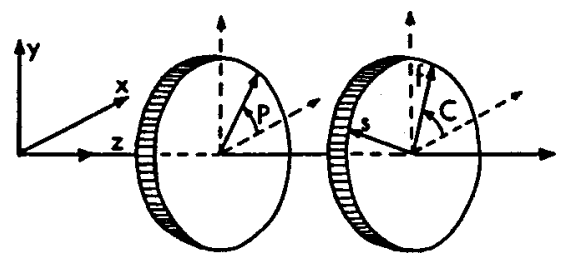

Fig. 1. Polarization-state generator that consists of a linear polarizer and linear retarder. $P$ and $C$ are the orientation angles of the transmission axis of the polarizer and the fast axis of the retarder, respectively, relative to the reference $x$ direction.

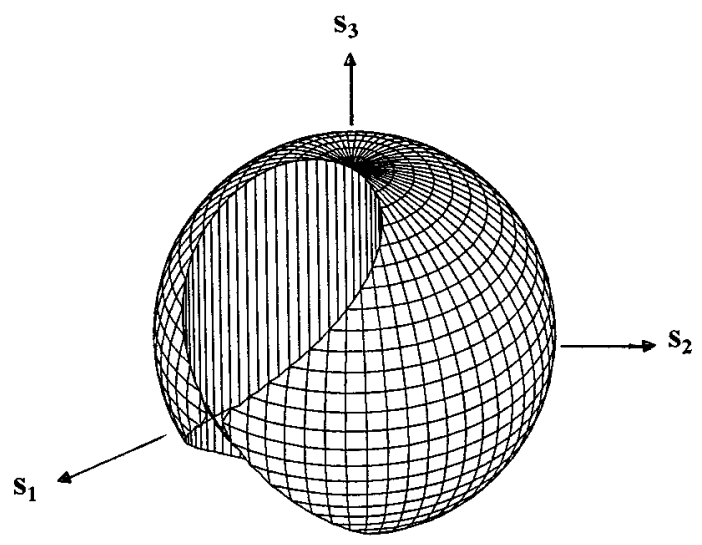

Fig. 2. Locus of the polarization state of light leaving a fixedpolarizer rotating-retarder system (Fig. 1) is represented by the line of intersection of a right-circular cylinder with the Poincare sphere. The diameter of the cylinder is half the diameter of the sphere for QWR $\left(\Delta=90^{\circ}\right)$.

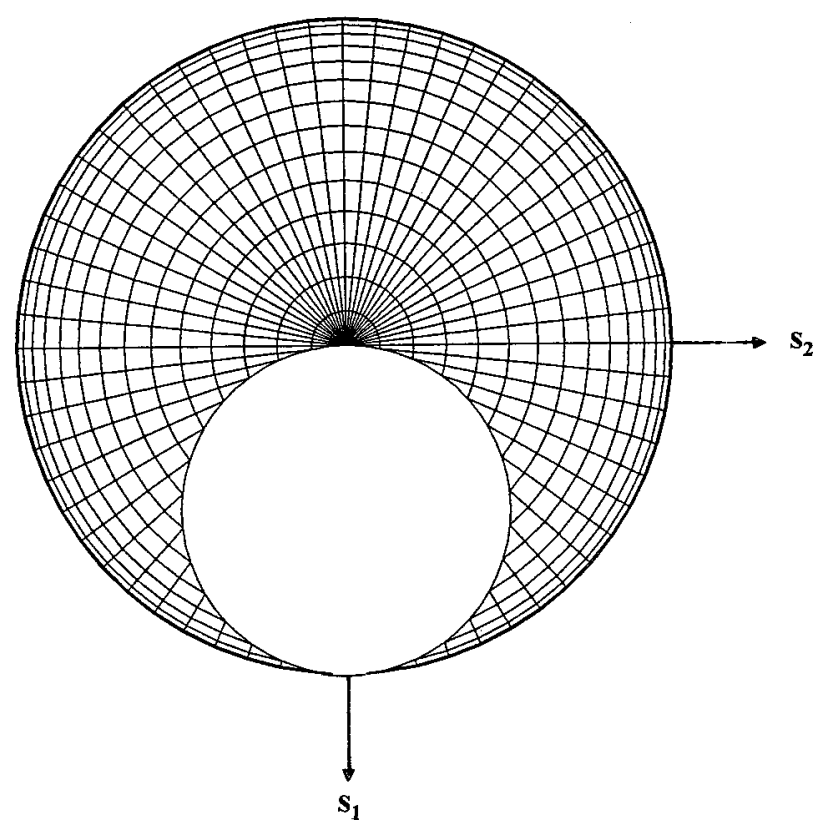

Fig. 3. Normal projection of Fig. 2 on the $\left(s_{1}, s_{2}\right)$ coordinate plane, where $\left(s_{1}, s_{2}, s_{3}\right)$ are the normalized Stokes parameters.

radius is half the sphere radius and the intersection contour passes through the north and south poles of the sphere, which correspond to the right- and left-circular polarizations, respectively. The grid of longitudes and latitudes on the Poincare sphere represent the equiazimuth and equiellipticity contours, respectively. ${ }^{3}$ It is in- teresting to note that the line of intersection represents the locus of all polarization states with equal azimuth and ellipticity angles, i.e., equal longitude and latitude on the sphere.

Figure 3 shows the normal projection of Fig. 2 in the equatorial $\left(s_{1}, s_{2}\right)$ plane. The circle of intersection of the cylinder with the equatorial plane is represented by Eq. (3), where $\Delta=90^{\circ}$.

For completeness, we also determine the two remaining projections of the trajectory of output states in the $\left(s_{1}, s_{3}\right)$ and $\left(s_{2}, s_{3}\right)$ coordinate planes. Elimination of $C$ between these normalized Stokes parameter pairs in Eqs. (1) gives

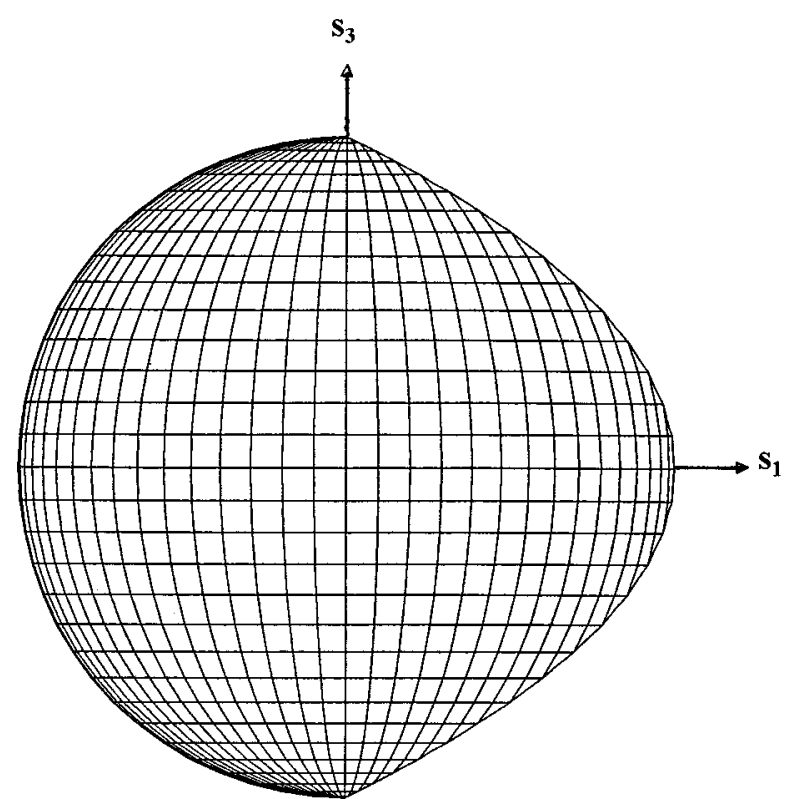

Fig. 4. Normal projection of Fig. 2 on the $\left(s_{1}, s_{3}\right)$ coordinate plane. The trajectory of the polarization states shown in Fig. 2 also lies on a parabolic cylinder parallel to the $s_{2}$ axis.

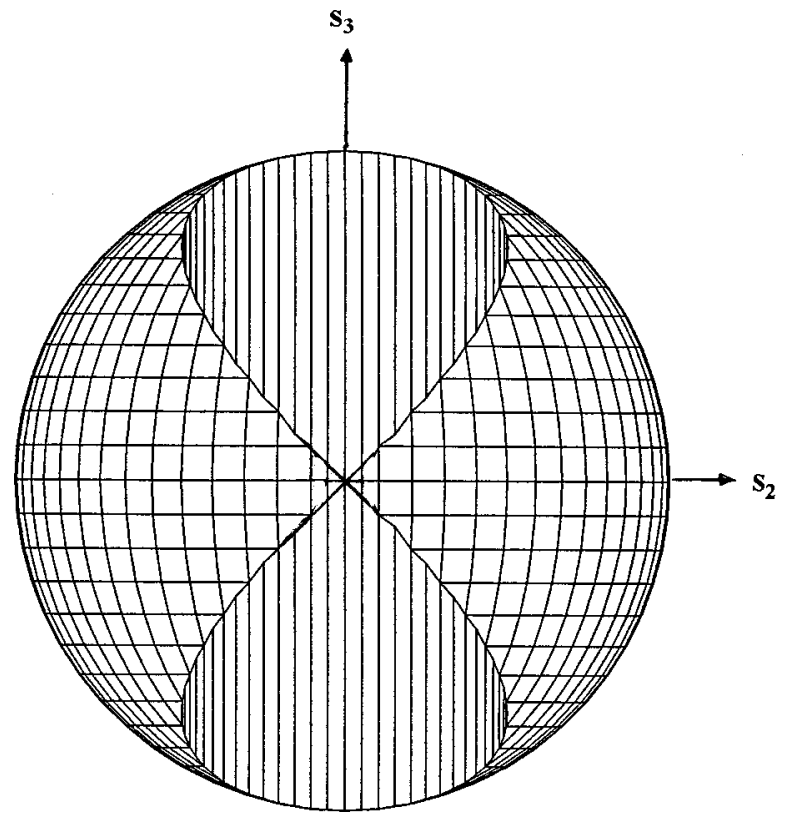

Fig. 5. Normal projection of Fig. 2 on the $\left(s_{2}, s_{3}\right)$ coordinate plane showing a head-on view of the figure- 8 contour. 


$$
\begin{gathered}
s_{3}{ }^{2}=\left[2 \cos ^{2}(\Delta / 2)\right]\left(1-s_{1}\right), \\
s_{3}{ }^{4}-\left(\sin ^{2} \Delta\right) s_{3}{ }^{2}+\left[4 \cos ^{2}(\Delta / 2)\right] s_{2}{ }^{2}=0 .
\end{gathered}
$$

Equation (6) shows another interesting result, namely, that the trajectory of output states is also the line of intersection of a parabolic cylinder parallel to the $s_{2}$ axis with the Poincaré sphere. Equation (7) is that of the projected figure-8 contour in the $\left(s_{2}, s_{3}\right)$ plane.

Figures 4 and 5 represent the two normal projections of Fig. 2 in the $\left(s_{1}, s_{3}\right)$ and $\left(s_{2}, s_{3}\right)$ coordinate planes, respectively, and show the projected parabola and figure- 8 contours for $\Delta=90^{\circ}$.

The foregoing results apply equally when the direction of propagation of light in Fig. 1 is reversed and the system functions as an elliptic analyzer or polarimeter. In this case the normalized Stokes parameters are those of the input elliptic polarization state of maximum transmission through the system for given orientations of the optical elements.

In conclusion, a detailed analysis of the fixed-polarizer rotating-retarder optical system has been presented. The three-dimensional figure-8 locus of (output or input) polarization states characteristic of this common system is shown to be the line of intersection of the Poincaré sphere with a right-circular cylinder whose axis is parallel to the polar axis of the sphere and whose radius is equal to the squared sine of half the retardance of the rotating retarder. These results complement those obtained by others ${ }^{8-10}$ concerning this locus.

\section{ACKNOWLEDGMENT}

I thank Paul Herrington for his assistance with the graphics.

The author's e-mail address is razzam@uno.edu.

\section{REFERENCES}

1. D. Clarke and J. F. Grainger, Polarized Light and Optical Measurement (Pergamon, New York, 1971).

2. R. M. A. Azzam, "Photopolarimetric measurement of the Mueller matrix by Fourier analysis of a single detected signal," Opt. Lett. 2, 148-150 (1978).

3. R. M. A. Azzam and N. M. Bashara, Ellipsometry and Polarized Light (North-Holland, Amsterdam, 1987).

4. P. S. Hauge, "Recent development in instrumentation in ellipsometry," Surf. Sci. 96, 108-140 (1980).

5. H. Poincaré, Theorie Mathématique de la Lumière (Gauthiers-Villars, Paris, 1892), Vol. II, Chap. 12.

6. H. G. Jerrard, "Transmission of light through birefringent and optically active media: the Poincare sphere," J. Opt. Soc. Am. 44, 634-640 (1954).

7. J. E. Bigelow and R. A. Kashnow, "Poincaré sphere analysis of liquid crystal optics," Appl. Opt. 16, 2090-2096 (1977).

8. D. S. Sabatke, M. R. Descour, E. L. Dereniak, W. C. Sweatt, S. A. Kemme, and G. S. Phipps, "Optimization of retardance for a complete Stokes polarimeter," Opt. Lett. 25, 802-804 (2000).

9. S. R. Rajagopalan and S. Ramaseshan, "Rotating elliptic analysers for the automatic analysis of polarised lightpart I," Proc. Indian Acad. Sci. Sect. A 60, 297-312 (1964).

10. R. M. A. Azzam, T. L. Bundy, and N. M. Bashara, "The fixed-polarizer nulling scheme in generalized ellipsometry," Opt. Commun. 7, 110-115 (1973). 